\title{
A Direct Retro-Reformatsky Fragmentation: Formal Ring Enlargement Transformation of Cyclic Ketones for Novel and Practical Synthesis of Heterocyclic Enamines
}

\author{
Mei-Xin Zhao, ${ }^{a}$ Mei-Xiang Wang, ${ }^{a}{ }^{*}$ Chu-Yi Yu, ${ }^{a}$ Zhi-Tang Huang ${ }^{a}$ and George W. J. Fleet ${ }^{b}$ \\ ${ }^{a}$ Laboratory of Chemical Biology, Center for Molecular Science, Institute of Chemistry, Chinese \\ Academy of Sciences, Beijing 100080, China. ${ }^{b}$ Dyson Perrins Laboratory, Department of Chemistry, \\ Oxford University, South Parks Road, Oxford, UK
}

mxwang@iccas.ac.cn

Content

1. General experimental information

2. Synthesis of the Reformatsky adducts 4

3. Spectral data of $\omega$-bromo- $\beta$-ketoesters 5

4. Spectral data of $\omega$-azido- $\beta$-ketoesters 7

5. Synthesis of the vicinal tricarbonyl derivatives $\mathbf{1 0}$

6. Reference

7. ${ }^{1} \mathrm{H}$ and ${ }^{13} \mathrm{C}$ NMR spectra of $6,8 \mathbf{c}$ and $8 d$

\section{General experimental information}

Melting points are uncorrected. Elemental analyses were performed at the Analytical Laboratory of the Institute. All chemicals were dired or purified according to standard procedures prior to use. 


\section{Synthesis of the Reformatsky adducts 4}

General Procedure for the Synthesis of the Reformatsky adducts 4. ${ }^{1}$ Trimethylcholrosilane $(0.3 \mathrm{~mL}, 24 \mathrm{mmol})$ was added from a syringe to a suspension of zinc powder $(2.1 \mathrm{~g}, 32 \mathrm{mmol})$ in anhydrous ethyl ether $(50 \mathrm{~mL})$. The mixture was stirred for $15 \mathrm{~min}$ at room temperature. Then the mixture was heated to reflux, and pure ethyl bromoacetate $(4 \mathrm{~g}, 24 \mathrm{mmol})$ and cyclic ketone (20 mmol) were added at such a rate that a gentle reflux was kept (20 min). After being heated to reflux for another $1 \mathrm{~h}$, the mixture was cooled and stirred for $15 \mathrm{~min}$ with $2 \mathrm{~N}$ hydrochloric acid $(100 \mathrm{~mL})$. The aqueous phase was extracted with ethyl ether $(2 \times 50 \mathrm{~mL})$ and the combined organic phase was washed with a $5 \% \mathrm{NaHCO}_{3}$ solution $(2 \times 50 \mathrm{~mL})$. After drying $\left(\mathrm{K}_{2} \mathrm{CO}_{3}\right)$, the hydroxy ester was chromatographyed on a silica gel column (petroleum ether: ethyl ether=35:1 as an eluent) to give the product 4 .

Ethyl 1-Hydroxycyclopentylacetate (4a): ${ }^{1}$ Colorless oil, yield $51 \% ;{ }^{1} \mathrm{H} \mathrm{NMR}\left(300 \mathrm{MHz}, \mathrm{CDCl}_{3}\right)$ $\delta 4.11(\mathrm{q}, J=7.2 \mathrm{~Hz}, 2 \mathrm{H}), 3.37(\mathrm{~s}, 1 \mathrm{H}), 2.53(\mathrm{~s}, 2 \mathrm{H}), 1.79-1.70(\mathrm{~m}, 4 \mathrm{H}), 1.58-1.48(\mathrm{~m}, 4 \mathrm{H}), 1.21(\mathrm{t}, J=$ $7.2 \mathrm{~Hz}, 3 \mathrm{H})$.

Ethyl 1-Hydroxycyclohexylacetate (4b) ${ }^{1}$ Colorless oil, yield 88\%; ${ }^{1} \mathrm{H}$ NMR $\left(300 \mathrm{MHz}, \mathrm{CDCl}_{3}\right) \delta$ $4.14(\mathrm{q}, J=7.2 \mathrm{~Hz}, 2 \mathrm{H}), 3.08$ (br., $1 \mathrm{H}), 2.44(\mathrm{~s}, 2 \mathrm{H}), 1.67-1.59(\mathrm{~m}, 4 \mathrm{H}), 1.59-1.34(\mathrm{~m}, 6 \mathrm{H}), 1.25(\mathrm{t}, J=$ $7.2 \mathrm{~Hz}, 3 \mathrm{H})$.

Ethyl 1-Hydroxy-(4-methylcyclohexyl)acetate $(\mathbf{4 c}):^{2}$ Colorless oil, yield $71 \% ;{ }^{1} \mathrm{H}$ NMR $\left(300 \mathrm{MHz}, \mathrm{CDCl}_{3}\right) \delta 4.17(\mathrm{q}, J=7.2 \mathrm{~Hz}, 2 \mathrm{H}), 3.09$ (br., $\left.1 \mathrm{H}\right), 2.42(\mathrm{~s}, 2 \mathrm{H}), 1.76-1.72(\mathrm{~m}, 2 \mathrm{H}), 1.47-1.34$ $(\mathrm{m}, 7 \mathrm{H}), 1.28(\mathrm{t}, J=7.2 \mathrm{~Hz}, 3 \mathrm{H}), 0.91(\mathrm{~d}, J=4.8 \mathrm{~Hz}, 3 \mathrm{H})$.

Ethyl 1-Hydroxy-(2,6-dimethylcyclohexyl)acetate (4d): ${ }^{3}$ Colorless oil, yield 61\%; ${ }^{1} \mathrm{H}$ NMR $\left(300 \mathrm{MHz}, \mathrm{CDCl}_{3}\right) \delta 4.14(\mathrm{q}, J=7.2 \mathrm{~Hz}, 2 \mathrm{H}), 2.66$ (br., $\left.1 \mathrm{H}\right), 2.64(\mathrm{~s}, 2 \mathrm{H}), 1.65-1.54(\mathrm{~m}, 3 \mathrm{H}), 1.43-1.31$ $(\mathrm{m}, 5 \mathrm{H}), 1.27(\mathrm{t}, J=7.2 \mathrm{~Hz}, 3 \mathrm{H}), 0.95(\mathrm{~d}, J=6.6 \mathrm{~Hz}, 6 \mathrm{H})$.

Ethyl 1-Hydroxycycloheptxylacetate (4e): ${ }^{4}$ Colorless oil, yield 86\%; ${ }^{1} \mathrm{H} \mathrm{NMR}\left(300 \mathrm{MHz}, \mathrm{CDCl}_{3}\right)$ 
$\delta 4.19(\mathrm{q}, J=7.2 \mathrm{~Hz}, 2 \mathrm{H}), 3.26-3.25$ (br., $1 \mathrm{H}), 2.49(\mathrm{~s}, 2 \mathrm{H}), 1.82-1.38(\mathrm{~m}, 12 \mathrm{H}), 1.29(\mathrm{t}, J=7.2 \mathrm{~Hz}, 3 \mathrm{H})$.

Ethyl 1-Hydroxy-(2-adamantyl)acetate (4f): White crystal, mp $46-47^{\circ} \mathrm{C}$; yield $97 \%$; ${ }^{1} \mathrm{H}$ NMR $\left(300 \mathrm{MHz}, \mathrm{CDCl}_{3}\right) \delta 4.06(\mathrm{q}, J=7.2 \mathrm{~Hz}, 2 \mathrm{H}), 3.54(\mathrm{~s}, 1 \mathrm{H}), 2.62(\mathrm{~s}, 2 \mathrm{H}), 2.20(\mathrm{~d}, J=12.3 \mathrm{~Hz}, 2 \mathrm{H})$, $1.68-1.58(\mathrm{~m}, 10 \mathrm{H}), 1.40(\mathrm{~d}, J=12.3 \mathrm{~Hz}, 2 \mathrm{H}), 1.17(\mathrm{t}, J=7.2 \mathrm{~Hz}, 3 \mathrm{H}) ;{ }^{13} \mathrm{C} \mathrm{NMR}\left(75 \mathrm{MHz}, \mathrm{CDCl}_{3}\right) \delta$ 173.1, 73.7, 60.3, 41.9, 38.0, 37.0, 34.3, 32.4, 27.0, 26.9, 13.9; IR (KBr) 3474, 1706, 1473, 1204cm ${ }^{-1}$; MS(EI) $m / z 238\left(\mathrm{M}^{+}, 2 \%\right), 220$ (86), 150 (71), 105 (50), 79 (100) Anal. Calcd for $\mathrm{C}_{14} \mathrm{H}_{22} \mathrm{O}_{3}$ : C, 70.56 ; H, 9.30. Found: C, 70.66; H, 9.41.

Ethyl 1-Hydroxycyclooctylacetate (4g): ${ }^{5}$ Colorless oil, yield $63 \% ;{ }^{1} \mathrm{H}$ NMR $\left(300 \mathrm{MHz}, \mathrm{CDCl}_{3},\right) \delta$ $4.13(\mathrm{q}, J=7.2 \mathrm{~Hz}, 2 \mathrm{H}), 3.36(\mathrm{~s}, 1 \mathrm{H}), 2.43(\mathrm{~s}, 2 \mathrm{H}), 1.86-1.78(\mathrm{~m}, 2 \mathrm{H}), 1.63-1.41(\mathrm{~m}, 12 \mathrm{H}), 1.24(\mathrm{t}, J=$ 7.2Hz, 3H).

Ethyl 1,2,3,4-Tetrahydro-1-hydroxy-1-naphthylacetate (4h): ${ }^{6}$ Yellow oil, yield $80 \% ;{ }^{1} \mathrm{H}$ NMR $\left(300 \mathrm{MHz}, \mathrm{CDCl}_{3}\right) \delta 7.59(\mathrm{~d}, J=7.2 \mathrm{~Hz}, 1 \mathrm{H}), 7.29-7.18(\mathrm{~m}, 2 \mathrm{H}), 7.11(\mathrm{~d}, J=7.2 \mathrm{~Hz}, 1 \mathrm{H}), 4.22(\mathrm{q}, J=$ 7.2Hz, 2H), 3.89 (br. 1H), 2.93-2.75 (m, 4H), 2.14-2.09 (m, 1H), 2.05-1.95 (m, 2H), 1.85-1.78 (m, $1 \mathrm{H}), 1.30(\mathrm{t}, J=7.2 \mathrm{~Hz}, 3 \mathrm{H})$.

\section{Spectral data of $\omega$-bromo- $\beta$-ketoesters 5}

Ethyl 7-Bromo-3-oxoheptanoate (5a): ${ }^{7}$ Colorless oil, yield 84\%; ${ }^{1} \mathrm{H} \mathrm{NMR}\left(300 \mathrm{MHz}, \mathrm{CDCl}_{3}\right) \delta$ $12.06(\mathrm{~s}, 0.1 \mathrm{H}), 4.93(\mathrm{~s}, 0.1 \mathrm{H}), 4.13(\mathrm{q}, J=7.2 \mathrm{~Hz}, 2 \mathrm{H}), 3.39(\mathrm{~s}, 1.8 \mathrm{H}), 3.35(\mathrm{t}, J=6.6 \mathrm{~Hz}, 2 \mathrm{H}), 2.54(\mathrm{t}$, $J=7.2 \mathrm{~Hz}, 1.8 \mathrm{H}), 2.18(\mathrm{t}, J=7.5 \mathrm{~Hz}, 0.2 \mathrm{H}), 1.86-1.64(\mathrm{~m}, 4 \mathrm{H}), 1.22(\mathrm{t}, J=7.2 \mathrm{~Hz}, 3 \mathrm{H})$.

Ethyl 8-Bromo-3-oxooctanoate (5b): Yellow oil, yield: 90\%; ${ }^{1} \mathrm{H}$ NMR $\left(300 \mathrm{MHz}, \mathrm{CDCl}_{3}\right) \delta 12.09$ (s, 0.09H), $4.96(\mathrm{~s}, 0.09 \mathrm{H}), 4.18(\mathrm{q}, J=7.2 \mathrm{~Hz}, 2 \mathrm{H}), 3.41(\mathrm{~s}, 1.82 \mathrm{H}), 3.39(\mathrm{t}, J=7.2 \mathrm{~Hz}, 2 \mathrm{H}), 2.55(\mathrm{t}, J$ $=7.2 \mathrm{~Hz}, 1.82 \mathrm{H}$ ), $2.20(\mathrm{t}, J=7.5 \mathrm{~Hz}, 0.18 \mathrm{H}$ ), 1.85 (quin, $J=7.3 \mathrm{~Hz}, 2 \mathrm{H}$ ), 1.62 (quin, $J=7.5 \mathrm{~Hz}$, 2H), 1.46 (quin, $J=7.0 \mathrm{~Hz}, 2 \mathrm{H}), 1.27(\mathrm{t}, J=7.2 \mathrm{~Hz}, 3 \mathrm{H}) ;{ }^{13} \mathrm{C} \mathrm{NMR}\left(75 \mathrm{MHz}, \mathrm{CDCl}_{3}\right) \delta 202.5,178.2$, $175.1,167.2,89.2,61.3,60.0,49.3,42.6,34.8,33.5,32.4,27.5,25.4,22.4,14.2,14.1$; IR(KBr) 1745 , $1716 \mathrm{~cm}^{-1}$; MS(EI) m/e 221, $219\left(\mathrm{M}^{+}-\mathrm{OC}_{2} \mathrm{H}_{5}, 1 \%\right), 185\left(\mathrm{M}^{+}-\mathrm{Br}, 18\right), 179$ (21), 177 (21), 130 (76), 69 
(100). HRMS cacld for $\mathrm{C}_{10} \mathrm{H}_{18} \mathrm{BrO}_{3}(\mathrm{M}+1)$ 267.0413, 265.0434. Found: 267.0416, 265.0436.

Ethyl 8-Bromo-6-methyl-3-oxooctanoate (5c): Colorless oil, yield 82\%; ${ }^{1} \mathrm{H}$ NMR (300MHz, $\left.\mathrm{CDCl}_{3}\right) \delta 12.13(\mathrm{~s}, 0.015 \mathrm{H}), 4.99(\mathrm{~s}, 0.015 \mathrm{H}), 4.18(\mathrm{q}, J=7.2 \mathrm{~Hz}, 2 \mathrm{H}), 3.45(\mathrm{~s}, 1.97 \mathrm{H}), 3.51-3.36(\mathrm{~m}$, 2H), 2.61-2.56 (m, 1.97H), 2.12-2.28 (m, 0.03H), 1.91-1.83 (m, 1H), 1.74-1.66 (m, 4H), 1.47-1.40 (m, $1 \mathrm{H}), 1.27(\mathrm{t}, J=7.2 \mathrm{~Hz}, 3 \mathrm{H}), 0.90(\mathrm{~d}, J=6.0 \mathrm{~Hz}, 3 \mathrm{H}) ;{ }^{13} \mathrm{C} \mathrm{NMR}\left(75 \mathrm{MHz}, \mathrm{CDCl}_{3}\right) \delta 202.3,178.3$, $172.3,166.8,88.7,60.9,59.6,48.9,40.0,39.3,32.4,32.1,31.5,30.9,30.5,29.3,18.3,14.0,13.8 ;$ IR (KBr) 1744, 1717 $\mathrm{cm}^{-1}$; MS(EI) m/z $193\left(\mathrm{M}^{+}-\mathrm{CH}_{2} \mathrm{CO}_{2} \mathrm{Et}, 17 \%\right), 191$ (21), 130 (100), 116 (26), 83 (49), 55 (100). HRMS cacld for $\mathrm{C}_{11} \mathrm{H}_{20} \mathrm{Br}_{3} \mathrm{O}_{3}(\mathrm{M}+1)$ 281.0570, 279.0590. Found: 281.0573, 279.0591.

Ethyl 7-Bromo-4-methyl-3-oxononanoate (5d): Colorless oil, yield 85\%; ${ }^{1} \mathrm{H}$ NMR $(300 \mathrm{MHz}$, $\left.\mathrm{CDCl}_{3}\right) \delta 12.16(\mathrm{~s}, 0.11 \mathrm{H}), 5.00(\mathrm{~s}, 0.11 \mathrm{H}), 4.18(\mathrm{q}, J=7.2 \mathrm{~Hz}, 2 \mathrm{H}), 3.50(\mathrm{~s}, 1.78 \mathrm{H}), 2.69-2.67(\mathrm{~m}$, $1 \mathrm{H}), 2.31-2.18(\mathrm{~m}, 0.11 \mathrm{H}), 1.78-1.68(\mathrm{~m}, 7 \mathrm{H}), 1.45-1.38(\mathrm{~m}, 3 \mathrm{H}), 1.27(\mathrm{t}, J=7.2 \mathrm{~Hz}, 3 \mathrm{H}), 1.14(\mathrm{~d}, J=$ $6.9 \mathrm{~Hz}, 3 \mathrm{H}) ;{ }^{13} \mathrm{C} \mathrm{NMR}\left(75 \mathrm{MHz}, \mathrm{CDCl}_{3}\right) \delta 205.9,181.9,172.6,166.9,87.9,60.9,59.7,51.2,51.0$, 47.5, 46.1, 40.7, 39.2, 33.1, 31.5, 26.2, 25.0, 17.9, 15.8, 13.9; IR (KBr) 1747, 1714cm-1 $\mathrm{cm}^{-1}$ (EI) $\mathrm{m} / \mathrm{z}$ $294\left(\mathrm{M}^{+}+2,1 \%\right), 292\left(\mathrm{M}^{+}, 1\right), 213\left(\mathrm{M}^{+}-\mathrm{Br}, 7\right), 144$ (100), 125 (13), 115 (20), 98 (36), 97 (39), 55 (63). HRMS cacld for $\mathrm{C}_{12} \mathrm{H}_{22} \mathrm{BrO}_{3}(\mathrm{M}+1)$ 295.0726, 293.0747. Found: 295.0725, 293.0748.

Ethyl 9-Bromo-3-oxononanoate (5e): ${ }^{8}$ Colorless oil, yield 93\%; ${ }^{1} \mathrm{H}$ NMR $\left(300 \mathrm{MHz}, \mathrm{CDCl}_{3}\right) \delta$ $12.15(\mathrm{~s}, 0.1 \mathrm{H}), 5.02(\mathrm{~s}, 0.1 \mathrm{H}), 4.24(\mathrm{q}, J=7.2 \mathrm{~Hz}, 2 \mathrm{H}), 3.47(\mathrm{~s}, 1.8 \mathrm{H}), 3.45(\mathrm{t}, J=6.6 \mathrm{~Hz}, 2 \mathrm{H}), 2.60(\mathrm{t}$, $J=7.2 \mathrm{~Hz}, 1.8 \mathrm{H}$ ), $2.26(\mathrm{t}, J=7.5 \mathrm{~Hz}, 0.2 \mathrm{H}), 1.90$ (quin, $J=7.0 \mathrm{~Hz}, 2 \mathrm{H}), 1.66$ (quin, $J=7.4 \mathrm{~Hz}, 2 \mathrm{H}$ ), 1.49 (quin, $J=7.6 \mathrm{~Hz}, 2 \mathrm{H}$ ), 1.37 (quin, $J=7.0 \mathrm{~Hz}, 2 \mathrm{H}$ ), $1.33(\mathrm{t}, J=7.2 \mathrm{~Hz}, 3 \mathrm{H}$ ).

\section{Spectral data of $\omega$-azido- $\beta$-ketoesters 7}

Ethyl 7-Azido-3-oxoheptanoate (7a): ${ }^{8}$ Colorless oil, yield $71 \% ;{ }^{1} \mathrm{H}$ NMR $\left(300 \mathrm{MHz}, \mathrm{CDCl}_{3}\right) \delta$ $12.12(\mathrm{~s}, 0.07 \mathrm{H}), 4.99(\mathrm{~s}, 0.07 \mathrm{H}),, 4.20(\mathrm{q}, J=7.2 \mathrm{~Hz}, 2 \mathrm{H}), 3.44(\mathrm{~s}, 1.86 \mathrm{H}), 3.29(\mathrm{t}, J=6.6 \mathrm{~Hz}, 2 \mathrm{H})$, $2.60(\mathrm{t}, J=6.6 \mathrm{~Hz}, 1.86 \mathrm{H}), 2.20(\mathrm{t}, J=7.5 \mathrm{~Hz}, 0.14 \mathrm{H}), 1.72-1.58(\mathrm{~m}, 4 \mathrm{H}), 1.28(\mathrm{t}, J=7.2 \mathrm{~Hz}, 3 \mathrm{H})$.

Ethyl 8-Azido-3-oxooctanoate (7b): ${ }^{8}$ Colorless oil, yield $89 \% ;{ }^{1} \mathrm{H}$ NMR $\left(300 \mathrm{MHz}, \mathrm{CDCl}_{3}\right) \delta$ 
$12.12(\mathrm{~s}, 0.07 \mathrm{H}), 4.98(\mathrm{~s}, 0.07 \mathrm{H}), 4.19(\mathrm{q}, J=7.2 \mathrm{~Hz}, 2 \mathrm{H}), 3.43(\mathrm{~s}, 1.86 \mathrm{H}), 3.27(\mathrm{t}, J=6.6 \mathrm{~Hz}, 2 \mathrm{H})$, $2.57(\mathrm{t}, J=7.2 \mathrm{~Hz}, 1.86 \mathrm{H}), 2.10(\mathrm{t}, J=7.5 \mathrm{~Hz}, 0.14 \mathrm{H}), 1.65-1.56(\mathrm{~m}, 4 \mathrm{H}), 1.43-1.35(\mathrm{~m}, 2 \mathrm{H}), 1.28(\mathrm{t}, J$ $=7.2 \mathrm{~Hz}, 3 \mathrm{H})$.

Ethyl 8-Azido-6-methyl-3-oxooctanoate (7c): Colorless oil, yield 72\%; ${ }^{1} \mathrm{H}$ NMR $(300 \mathrm{MHz}$, $\left.\mathrm{CDCl}_{3}\right) \delta 12.04(\mathrm{~s}, 0.1 \mathrm{H}), 4.89(\mathrm{~s}, 0.1 \mathrm{H}), 4.09(\mathrm{q}, J=7.2 \mathrm{~Hz}, 2 \mathrm{H}), 3.35(\mathrm{~s}, 1.8 \mathrm{H}), 3.30-3.16(\mathrm{~m}, 2 \mathrm{H})$, 2.51-2.45 (m, 1.8H), 2.15-2.05 (m, 0.2H), 1.56-1.48 (m, 3H), 1.37-1.31 (m, 2H), $1.18(\mathrm{t}, J=7.2 \mathrm{~Hz}$ $3 \mathrm{H}), 0.81(\mathrm{~d}, J=6.3 \mathrm{~Hz}, 3 \mathrm{H}) ;{ }^{13} \mathrm{C} \mathrm{NMR}\left(75 \mathrm{MHz}, \mathrm{CDCl}_{3}\right) \delta 202.6,178.5,172.6,167.1,89.0,61.2$, 59.8, 49.1 $40.3,35.3,33.0,32.4,29.8,29.7,18.9,14.1,14.0 ; \mathrm{IR}(\mathrm{KBr}) 2098,1743,1717 \mathrm{~cm}^{-1} ; \mathrm{MS}(\mathrm{EI})$ m/e $196\left(\mathrm{M}^{+}-\mathrm{OC}_{2} \mathrm{H}_{5}, 2 \%\right), 130$ (27), 115 (18), 98 (17), 84 (32), 70 (44), 55 (72), 43 (100). HRMS cacld for $\mathrm{C}_{11} \mathrm{H}_{20} \mathrm{~N}_{3} \mathrm{O}_{3}(\mathrm{M}+1)$ 242.1499. Found: 242.1501.

Ethyl 8-Azido-4-methyl-3-oxononanoate (7d). Yellow oil, yield 81\%; ${ }^{1} \mathrm{H}$ NMR $\left(300 \mathrm{MHz}, \mathrm{CDCl}_{3}\right)$ $\delta 12.06(\mathrm{~s}, 0.2 \mathrm{H}), 4.89(\mathrm{~s}, 0.2 \mathrm{H}), 4.10(\mathrm{q}, J=7.2 \mathrm{~Hz}, 2 \mathrm{H}), 3.40(\mathrm{~s}, 1.6 \mathrm{H}), 3.40-3.35(\mathrm{~m}, 1 \mathrm{H}), 2.61-2.54$ $(\mathrm{m}, 1 \mathrm{H}), 2.16-2.14(\mathrm{~m} 0.4 \mathrm{H}), 1.64-1.51(\mathrm{~m}, 1 \mathrm{H}), 1.50-1.23(\mathrm{~m}, 5 \mathrm{H}), 1.23-1.19(\mathrm{~m}, 3 \mathrm{H}), 1.17(\mathrm{t}, J=$ 7.2Hz, 3H), $1.04(\mathrm{~d}, J=6.6 \mathrm{~Hz}, 3 \mathrm{H}) ;{ }^{13} \mathrm{C} \mathrm{NMR}\left(75 \mathrm{MHz}, \mathrm{CDCl}_{3}\right) \delta 206.0,181.9,172.7,167.0,87.9$, 61.0, 59.7, 57.5, 47.5, 46.1, 39.2, 35.9, 33.5, 32.0, 23.5, 19.1, 17.8, 15.8, 14.0; IR(KBr) 2106, 1747, $1714 \mathrm{~cm}^{-1} ; \mathrm{MS}(\mathrm{EI}) \mathrm{m} / \mathrm{z} 213\left(\mathrm{M}^{+}-\mathrm{N}_{3}, 34 \%\right), 182$ (44), 144 (92), 115 (78), 111 (98), 95 (100), 67 (100). HRMS cacld for $\mathrm{C}_{12} \mathrm{H}_{22} \mathrm{~N}_{3} \mathrm{O}_{3}(\mathrm{M}+1)$ 256.1656. Found: 256.1657.

Ethyl 9-Azido-3-oxononanoate (7f): ${ }^{8}$ Colorless oil, yield $89 \% ;{ }^{1} \mathrm{H}$ NMR $\left(300 \mathrm{MHz}, \mathrm{CDCl}_{3}\right) \delta$ $12.10(\mathrm{~s}, 0.1 \mathrm{H}), 4.96(\mathrm{~s}, 0.1 \mathrm{H}), 4.18(\mathrm{q}, J=7.2 \mathrm{~Hz}, 2 \mathrm{H}), 3.42(\mathrm{~s}, 1.8 \mathrm{H}), 3.25(\mathrm{t}, J=6.6 \mathrm{~Hz}, 2 \mathrm{H}), 2.54(\mathrm{t}$, $J=7.2 \mathrm{~Hz}, 1.8 \mathrm{H}), 2.20(\mathrm{t}, J=7.5 \mathrm{~Hz}, 0.2 \mathrm{H}), 1.62-1.56(\mathrm{~m}, 4 \mathrm{H}), 1.39-1.31(\mathrm{~m}, 4 \mathrm{H}), 1.27(\mathrm{t}, J=7.2 \mathrm{~Hz}$ $3 \mathrm{H})$.

5. Synthesis of the Vicinal Tricarbonyl Derivatives $10{ }^{9}$ A solution of (fused) sodium acetate (270 mg, $3.3 \mathrm{mmol})$ in hot glacial acetic acid $(5 \mathrm{~mL})$ was prepared in a $25 \mathrm{~mL}$ round-bottomed flask. Tri-bromo compound $\mathbf{6 b}$ ( $422 \mathrm{mg}, 1 \mathrm{mmol}$ ) was added, and the mixture was heated to boiling until the 
precipitation of sodium bromide ceases $(\sim 3 \mathrm{~h})$. The mixture was cooled to room temperature, and then the precipitated salt was removed by a short silica gel column chromatography and washed by ethyl acetate. Combination of the eluent and evaporation of the solvent under the reduced pressure gave the crude product which was chromatographed on silica gel with ethyl acetate-petrulem ether mixture to afford the vicinal tricarbonyl derivatives $\mathbf{1 0}$.

Ethyl 2-Acetoxy-8-bromo-2-hydroxy-3-oxooctanoate (8). Yellow oil, yield 45\%; ${ }^{1} \mathrm{H}$ NMR $\left(300 \mathrm{MHz}, \mathrm{CDCl}_{3}\right) \delta 5.47(\mathrm{~s}, 1 \mathrm{H}), 4.26(\mathrm{q}, J=7.2 \mathrm{~Hz}, 2 \mathrm{H}), 3.38(\mathrm{t}, J=6.9 \mathrm{~Hz}, 2 \mathrm{H}), 2.67(\mathrm{t}, J=6.9 \mathrm{~Hz}$, 2H), $2.21(\mathrm{~s}, 3 \mathrm{H}), 1.84$ (quin, $J=7.2 \mathrm{~Hz}, 2 \mathrm{H}$ ), 1.62 (quin, $J=7.6 \mathrm{~Hz}, 2 \mathrm{H}$ ), 1.43 (quin, $J=7.0 \mathrm{~Hz}, 2 \mathrm{H}$ ), $1.29(\mathrm{t}, J=7.2 \mathrm{~Hz}, 3 \mathrm{H}) ;{ }^{13} \mathrm{C} \mathrm{NMR}\left(75 \mathrm{MHz}, \mathrm{CDCl}_{3}\right) \delta 199.5,169.3,164.3,77.3,62.3,39.3,33.1,32.1$, 27.2, 21.9, 20.2, 13.8; IR (KBr) 3448, 1751, 1730, 1373, 1214 $\mathrm{cm}^{-1}$; MS (EI) 282(1\%), 280 (1), 179 (25), 177 (26), 146 (25), 104 (34), 69 (100), 43(88). HRMS cacld for $\mathrm{C}_{12} \mathrm{H}_{21} \mathrm{O}_{5} \mathrm{Br}(\mathrm{M}+1)$ 325.0468, 323.0488. Found: $325.0468,323.0487$.

\section{Reference}

1. Picotin, G,; Miginiac, P. J. Org. Chem. 1987, 52, 4796.

2. Regnier, G.; Canevan, R.; Le Douarec, J. C. Chim. Ther. 1969, 4, 174.

3. Duhaime, R. M.; Lombardo, D. A.; Skinner, I. A.; Weedon, A. C. J. Org. Chem. 1985, 50, 873.

4. Smith, A. B. III; Toder, B. H.; Branca, S. J.; Dieck, R. K. J. Am. Chem. Soc., 1981, 103, 1996.

5. Paquette, L. A.; Henzel, K. A. J. Am. Chem. Soc., 1975, 97, 4649.

6. Hafez, A.; Campbell, N. J. Org. Chem. 1960, 4115.

7. Holmquist, C. R.; Roskamp, E. J. Tetrahedron Lett. 1990, 31, 4991.

8. Lambert, P. H.; Vaultier, M.; Carrie, R. J. Org. Chem. 1985, 50, 5352.

9. Mahran, M. R.; Abdou, W. M.; Sidky, M. M.; Wamhoff, H. Synthesis, 1987, 506. 


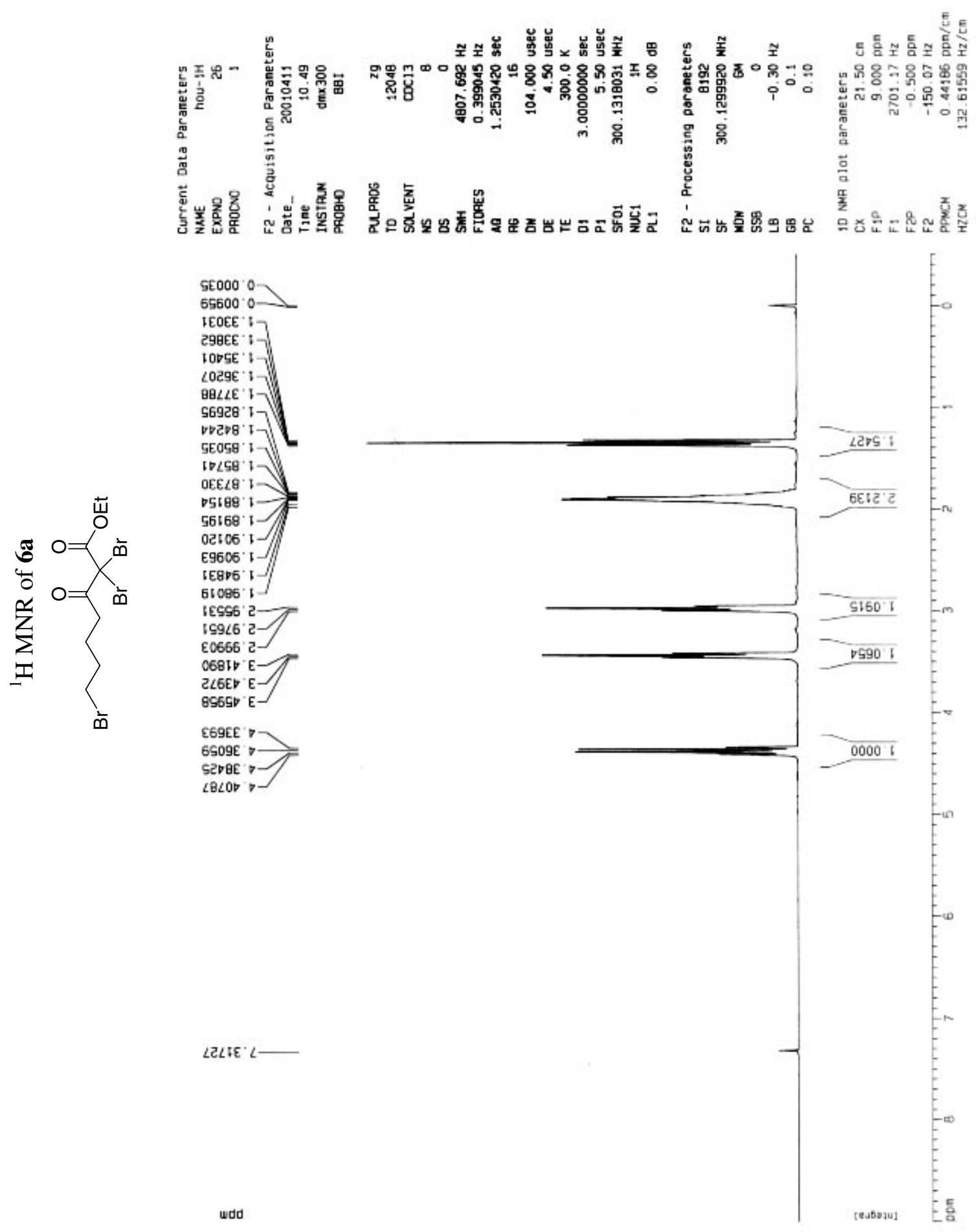



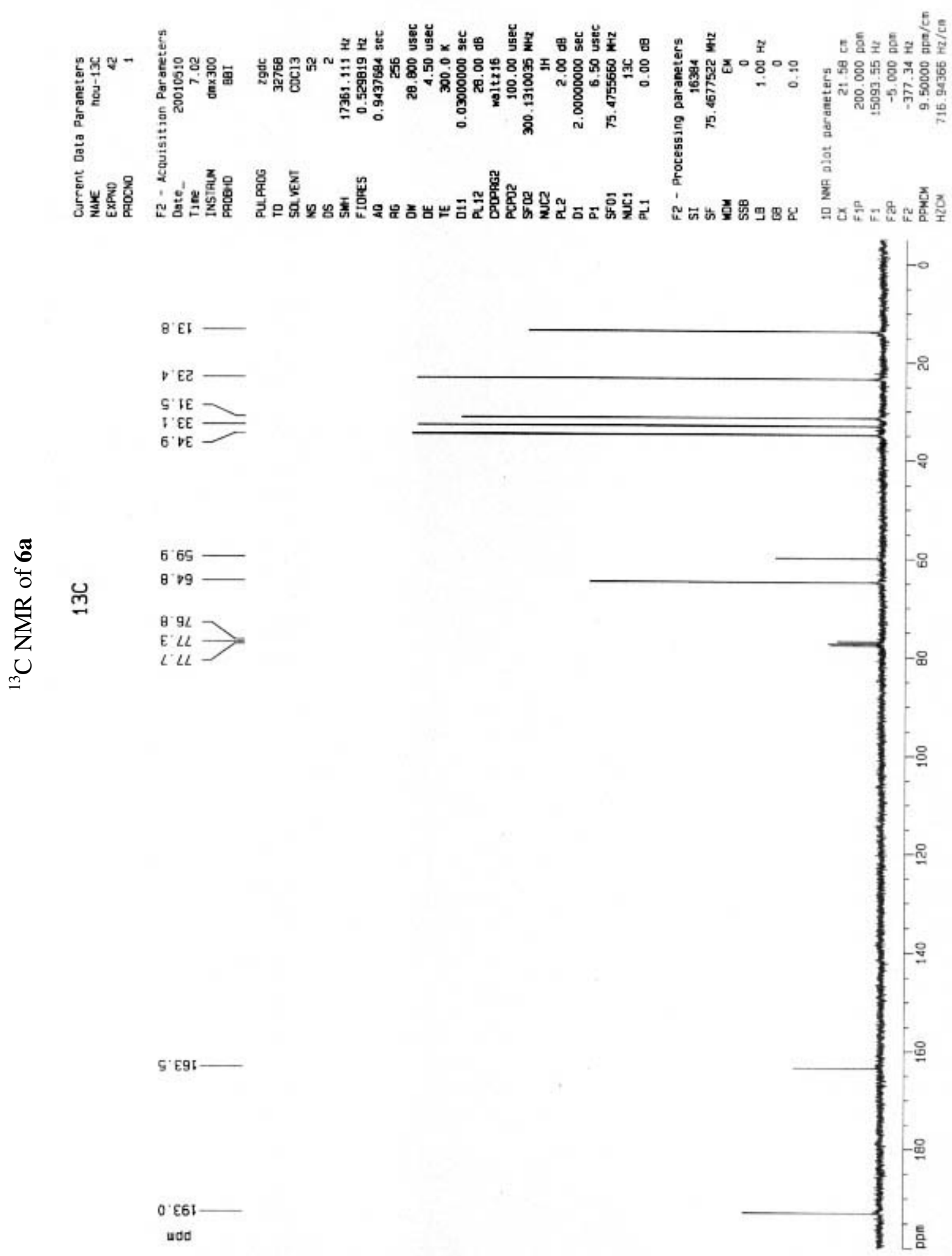

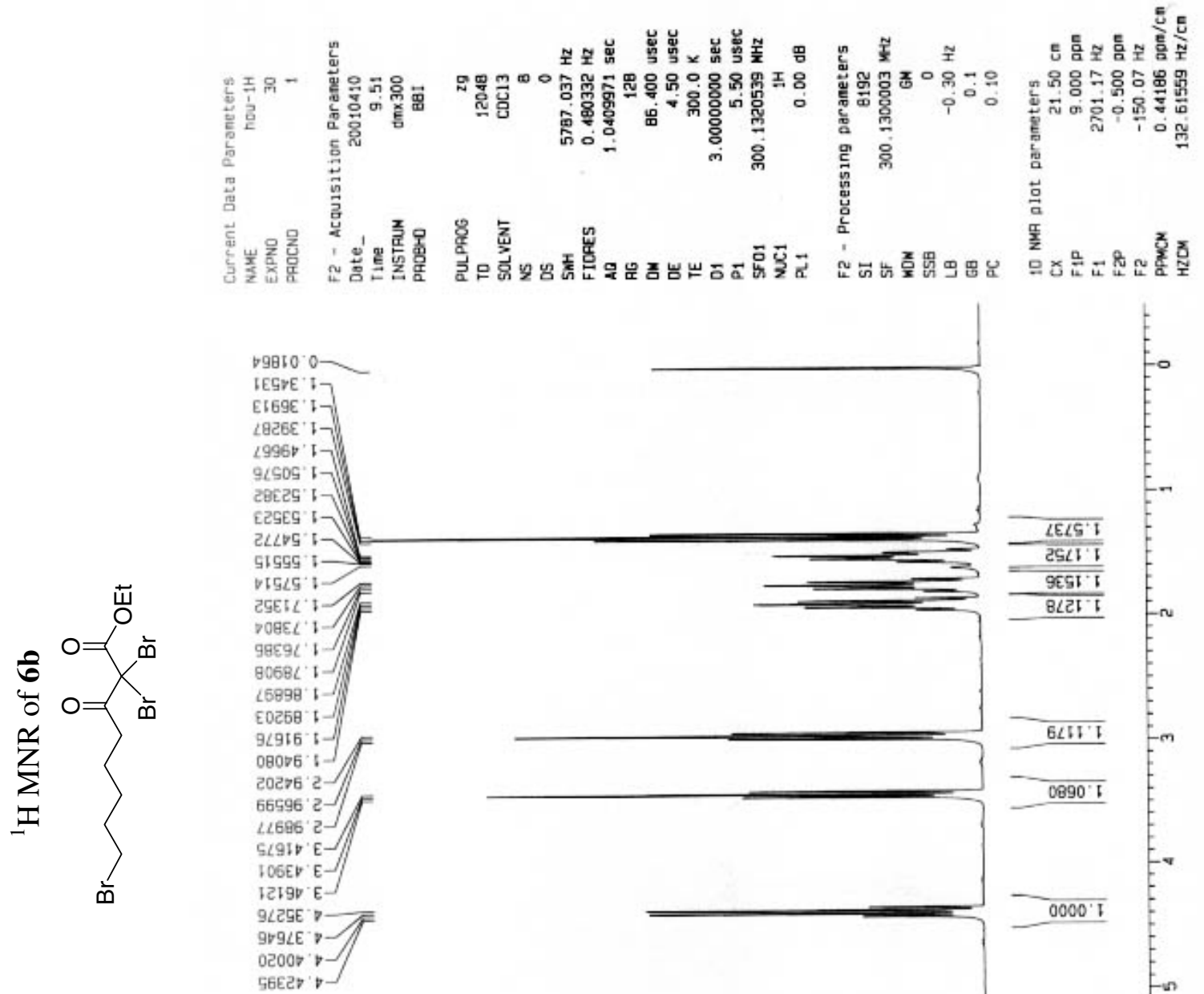

$\varepsilon \angle 682<-$
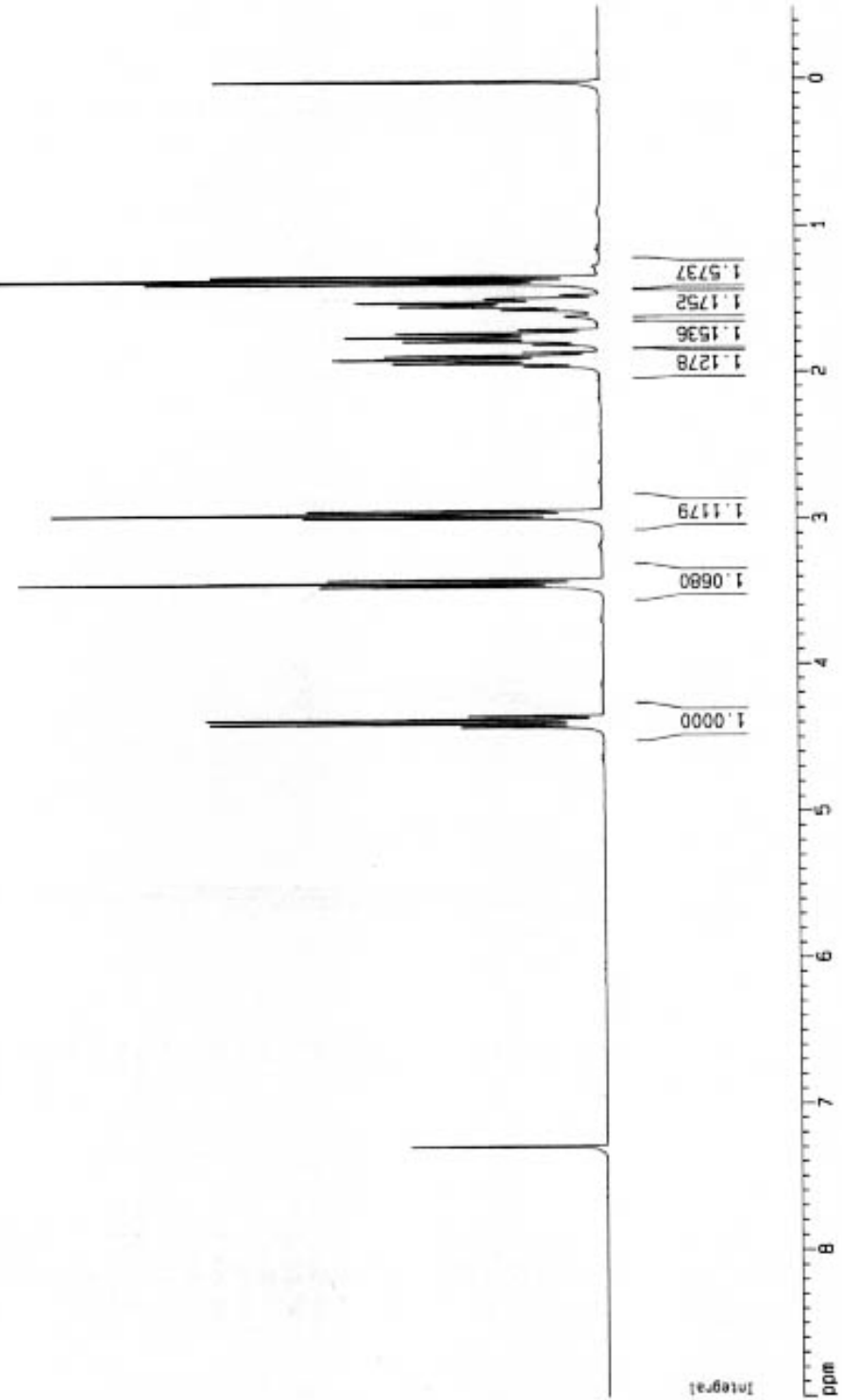


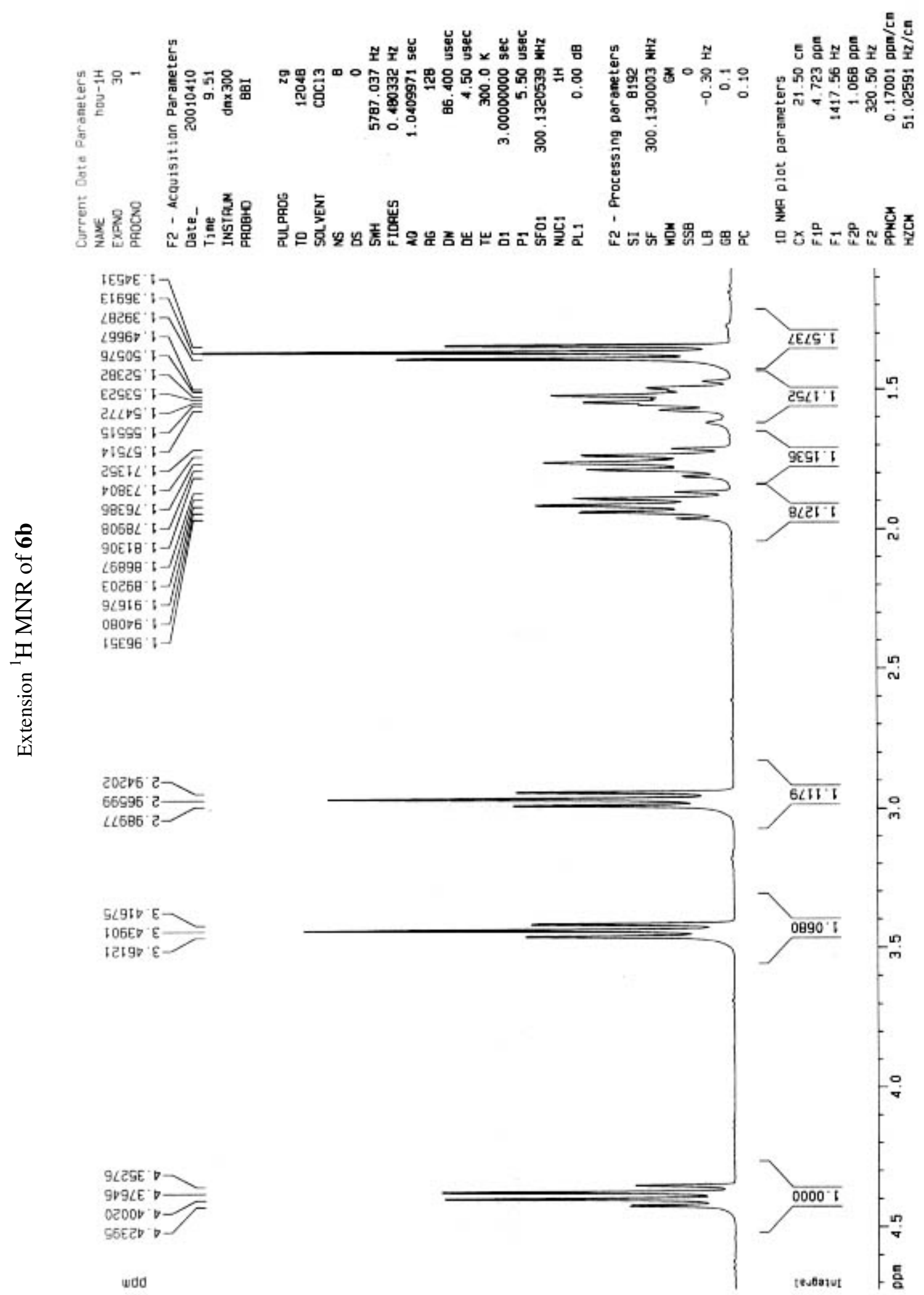



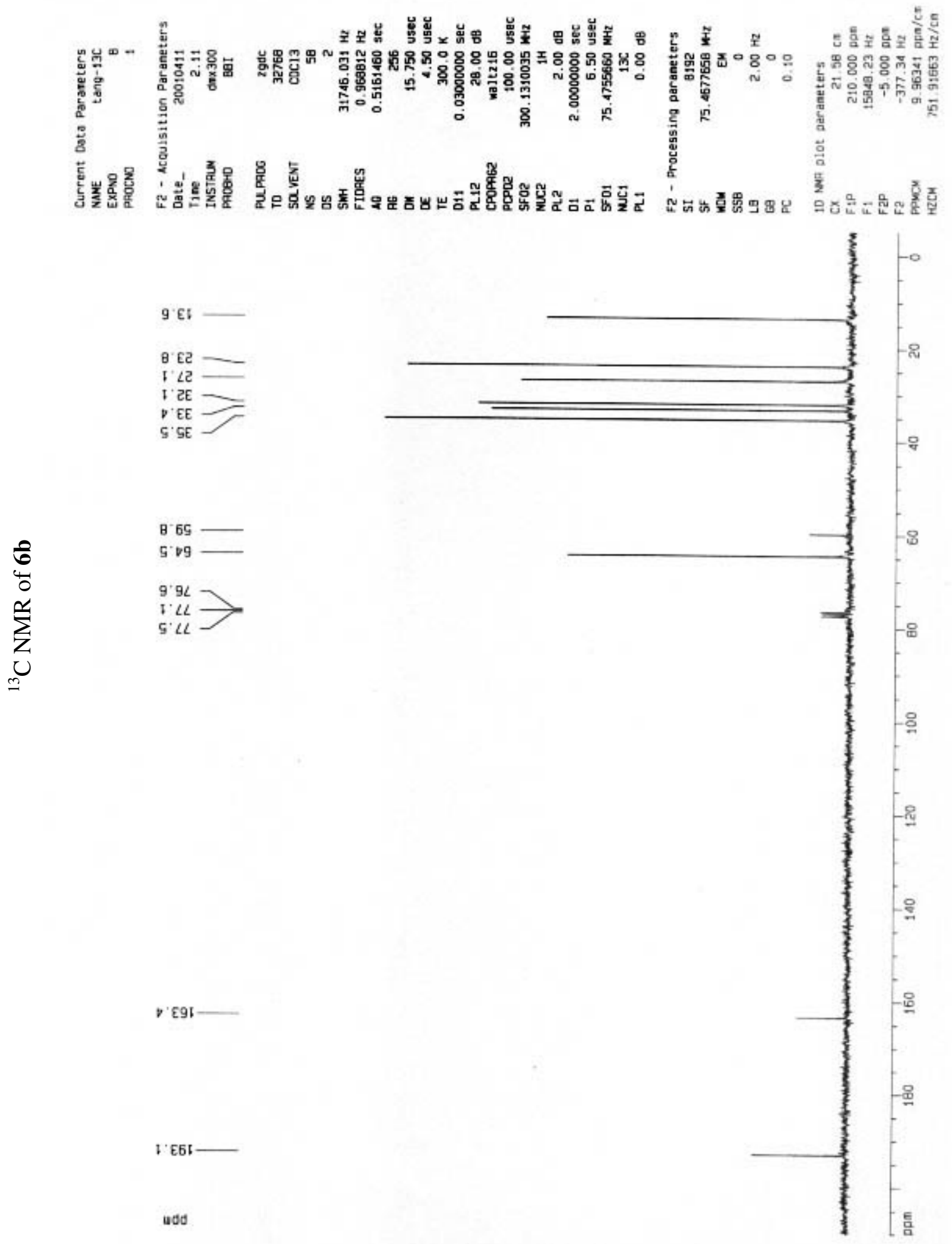

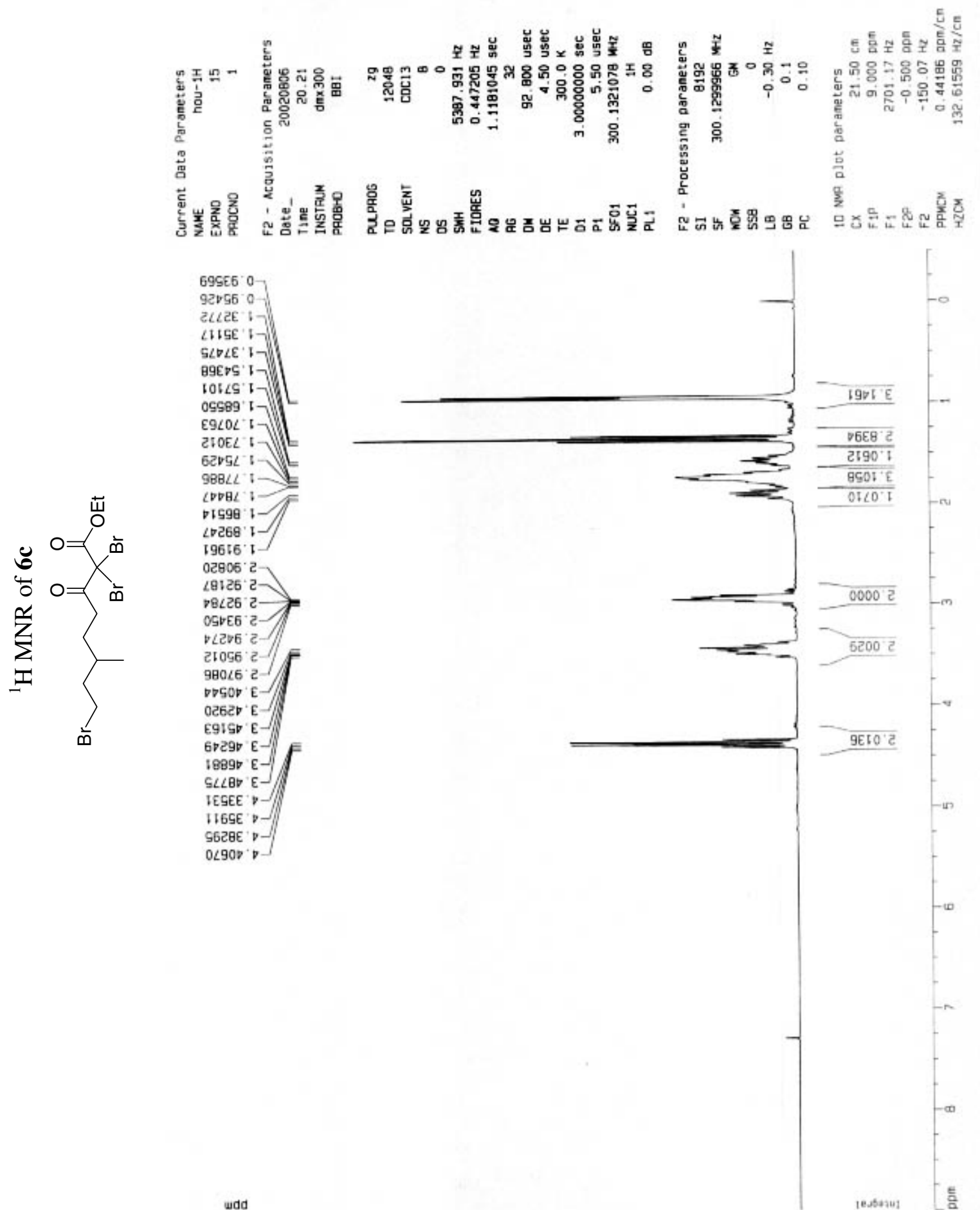

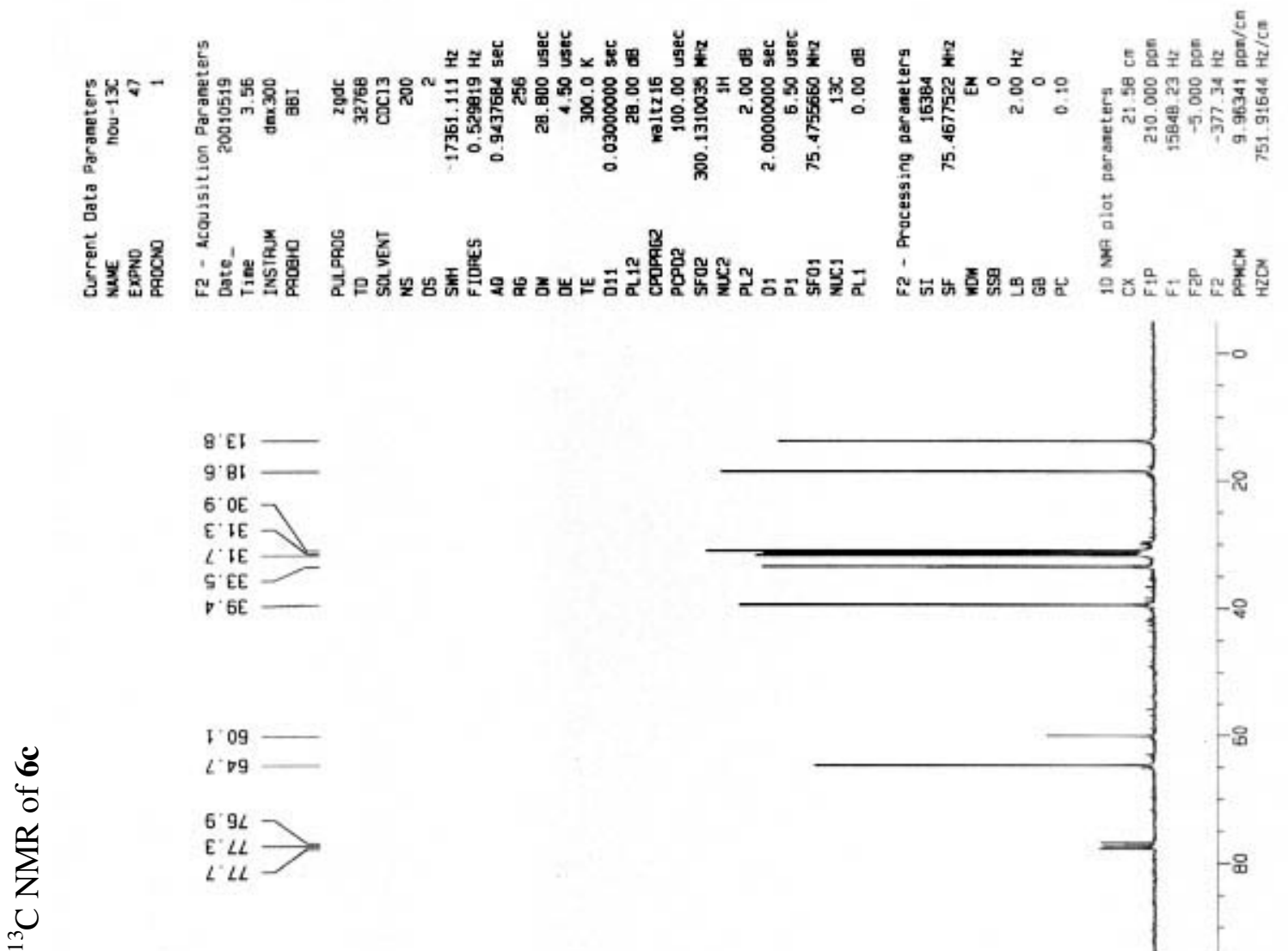

与. $E 95$

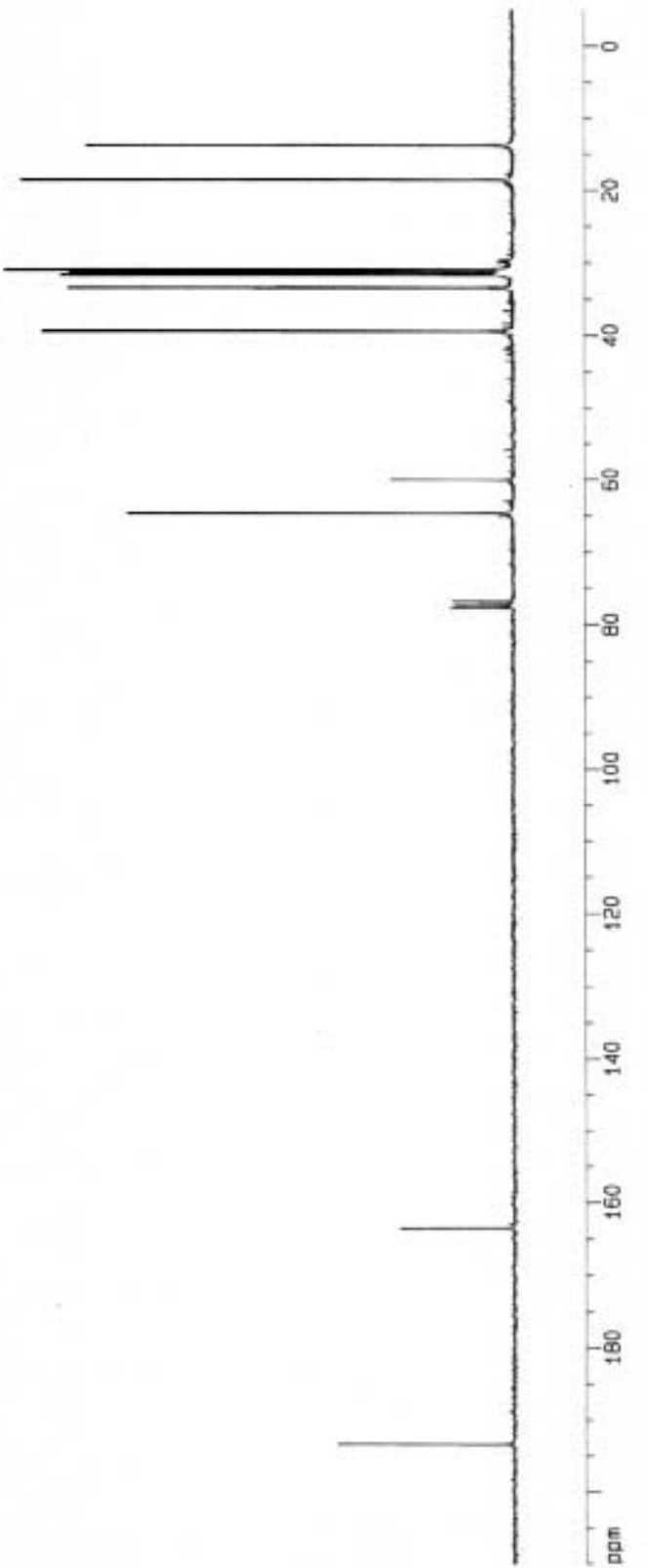



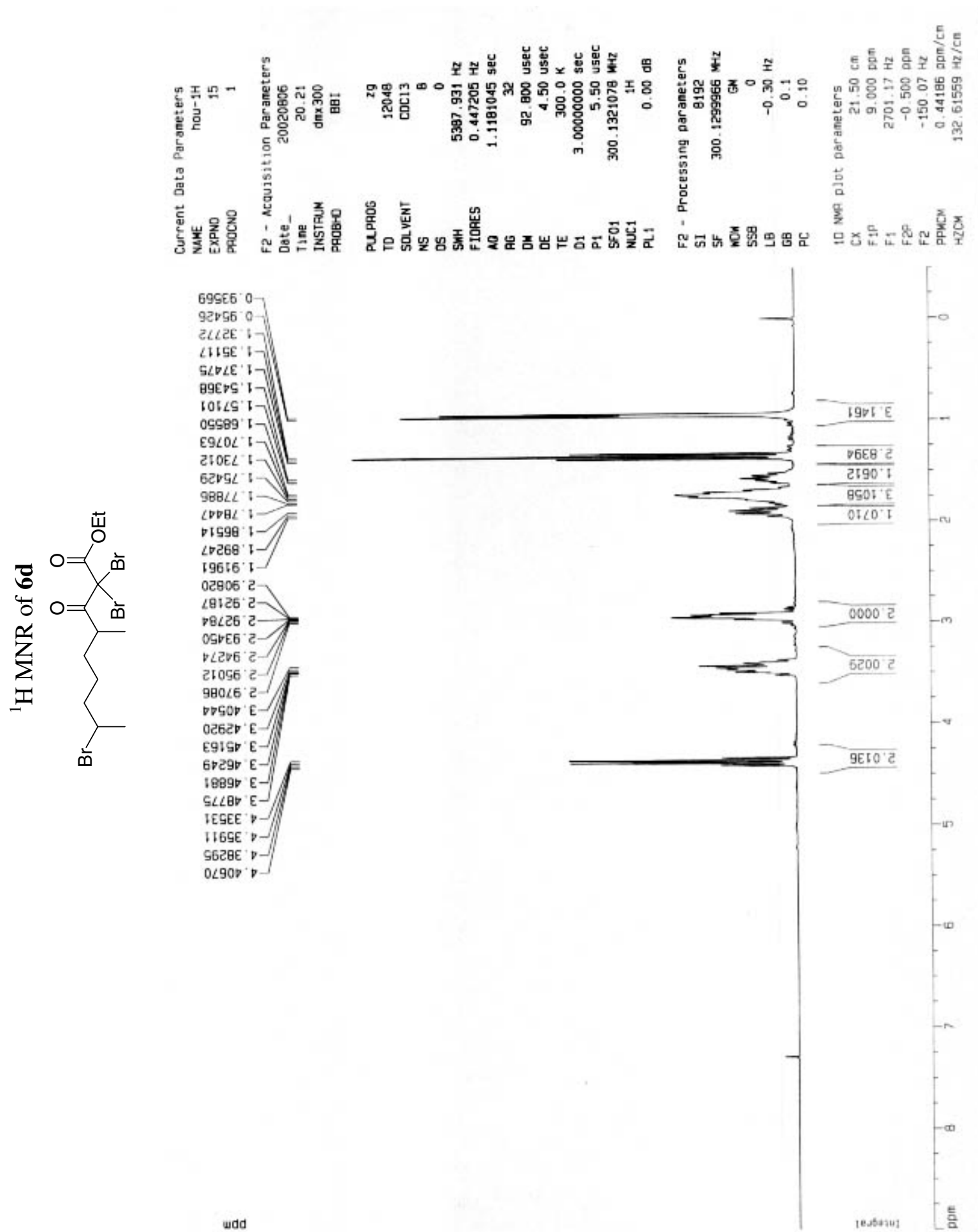

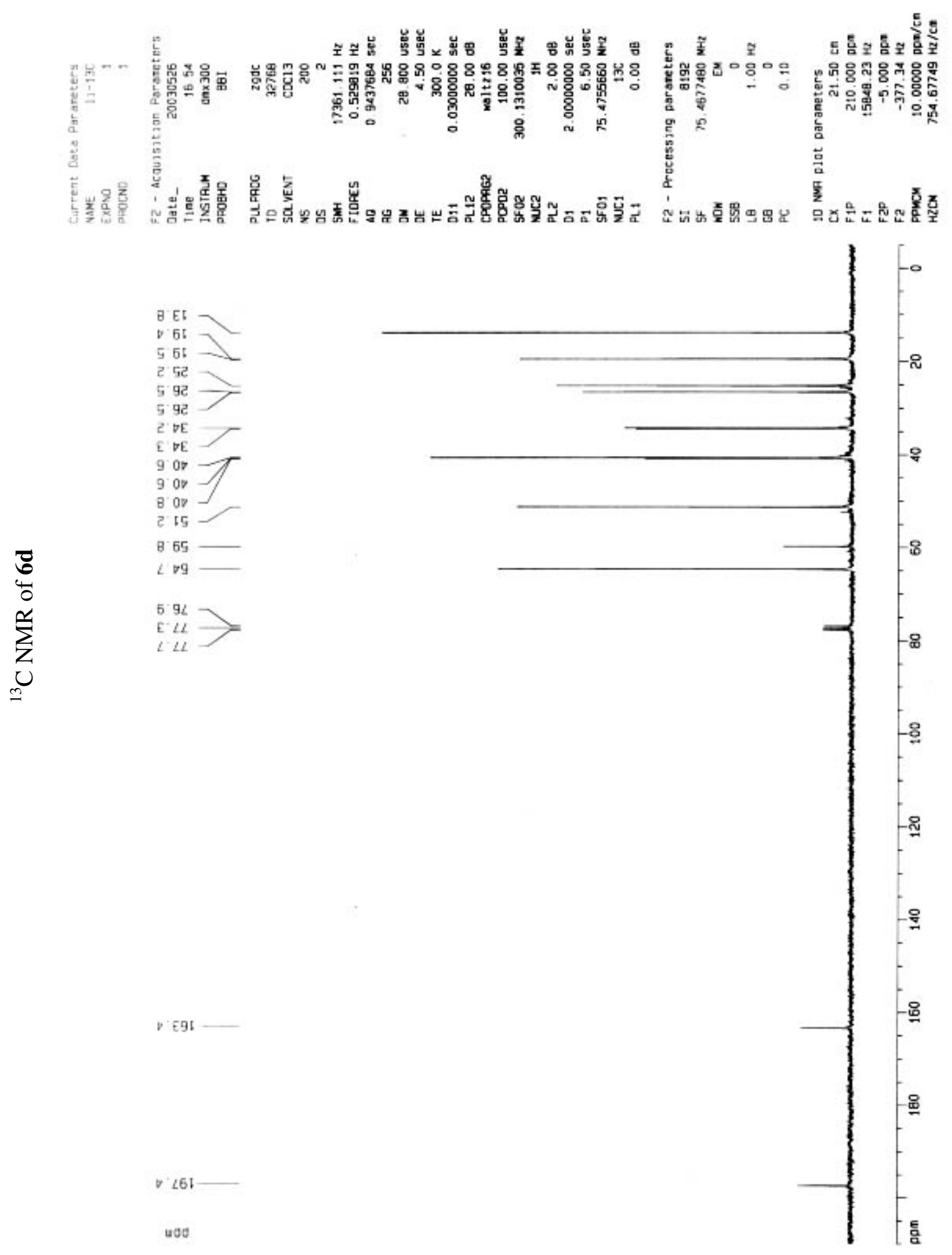

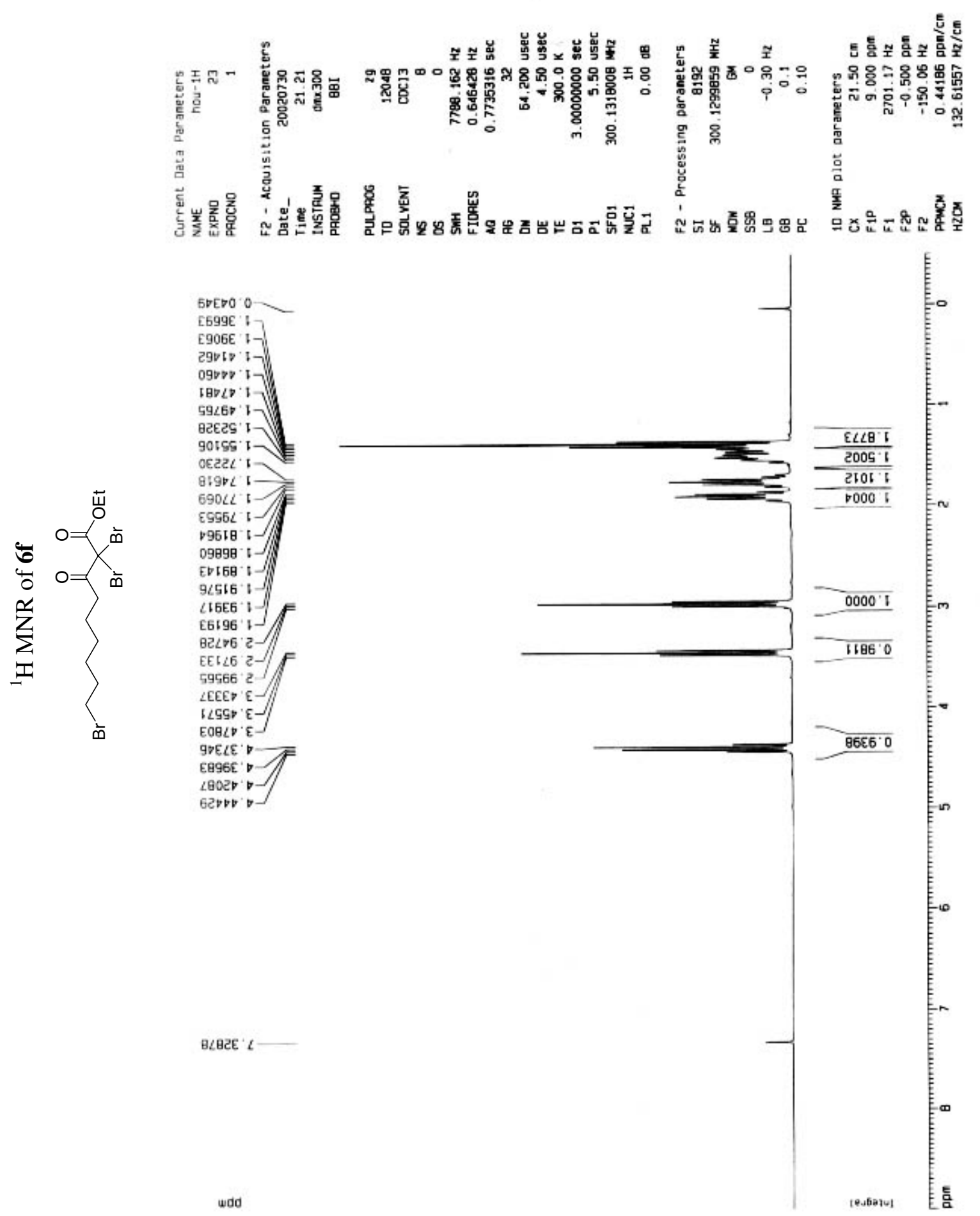

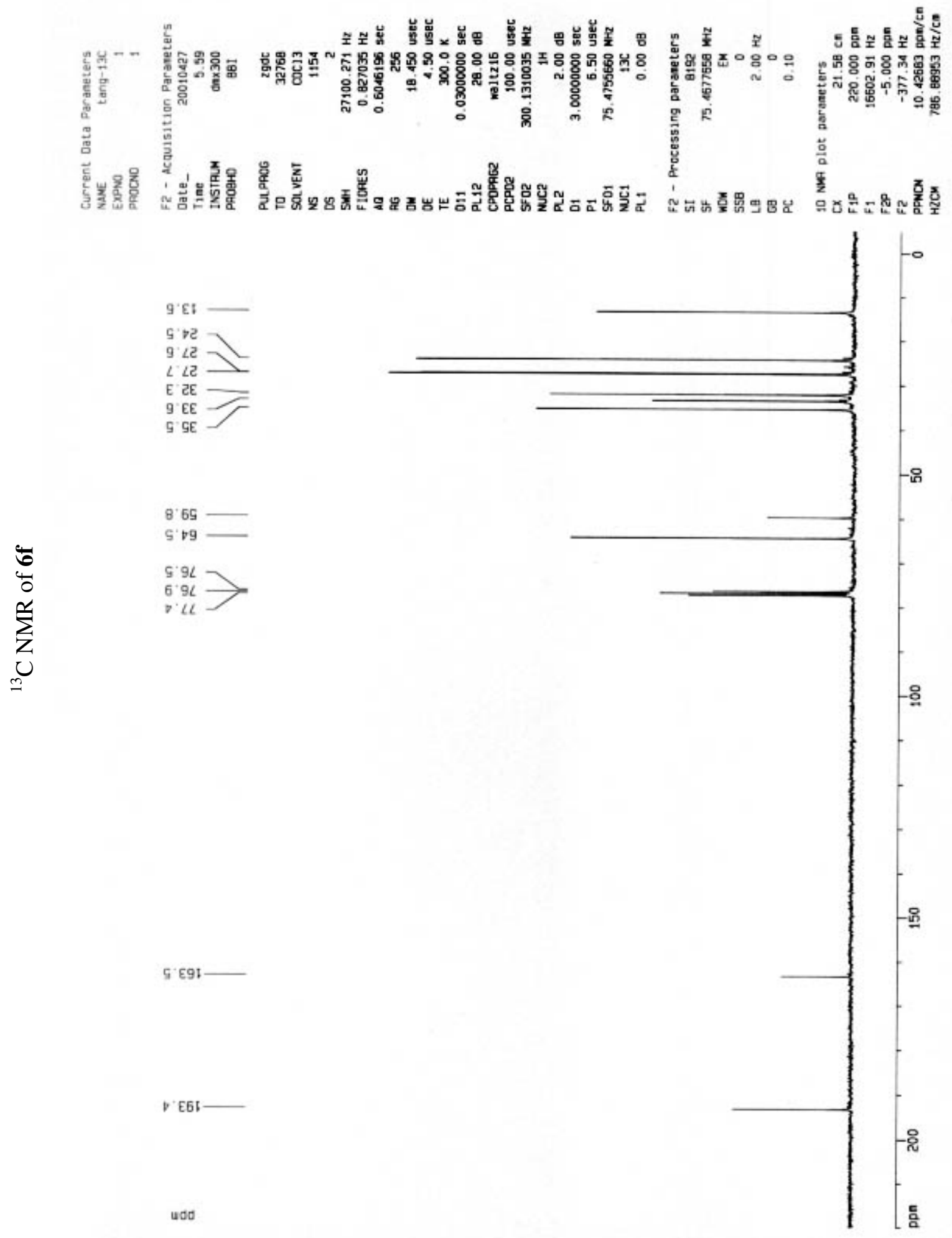

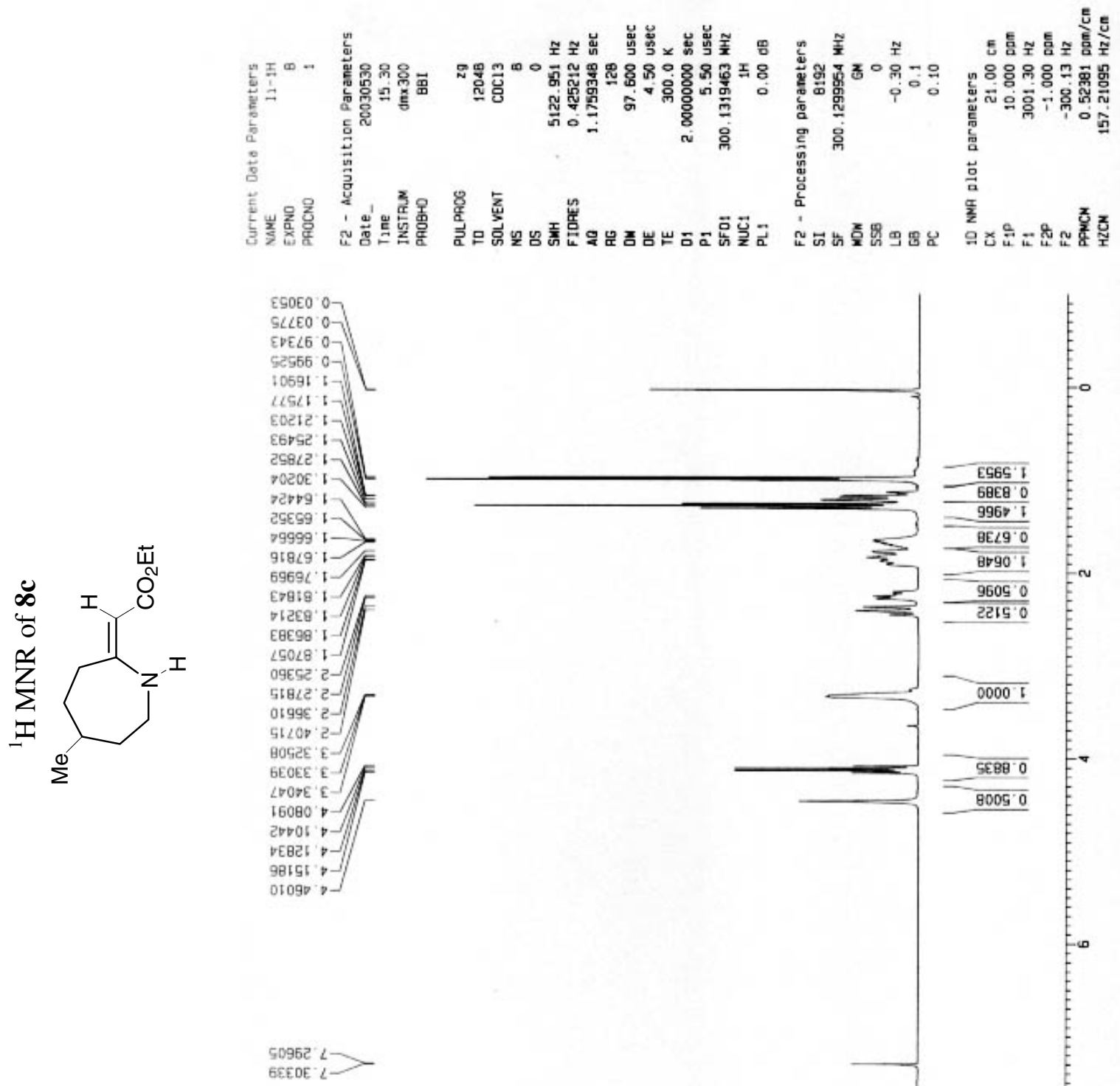

$B E \angle 9^{\prime} 8$

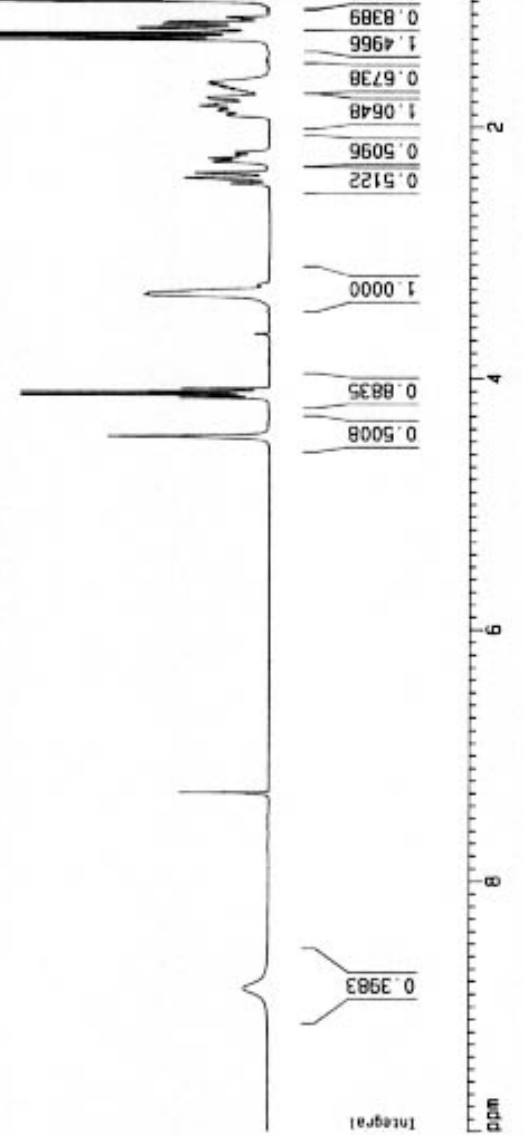



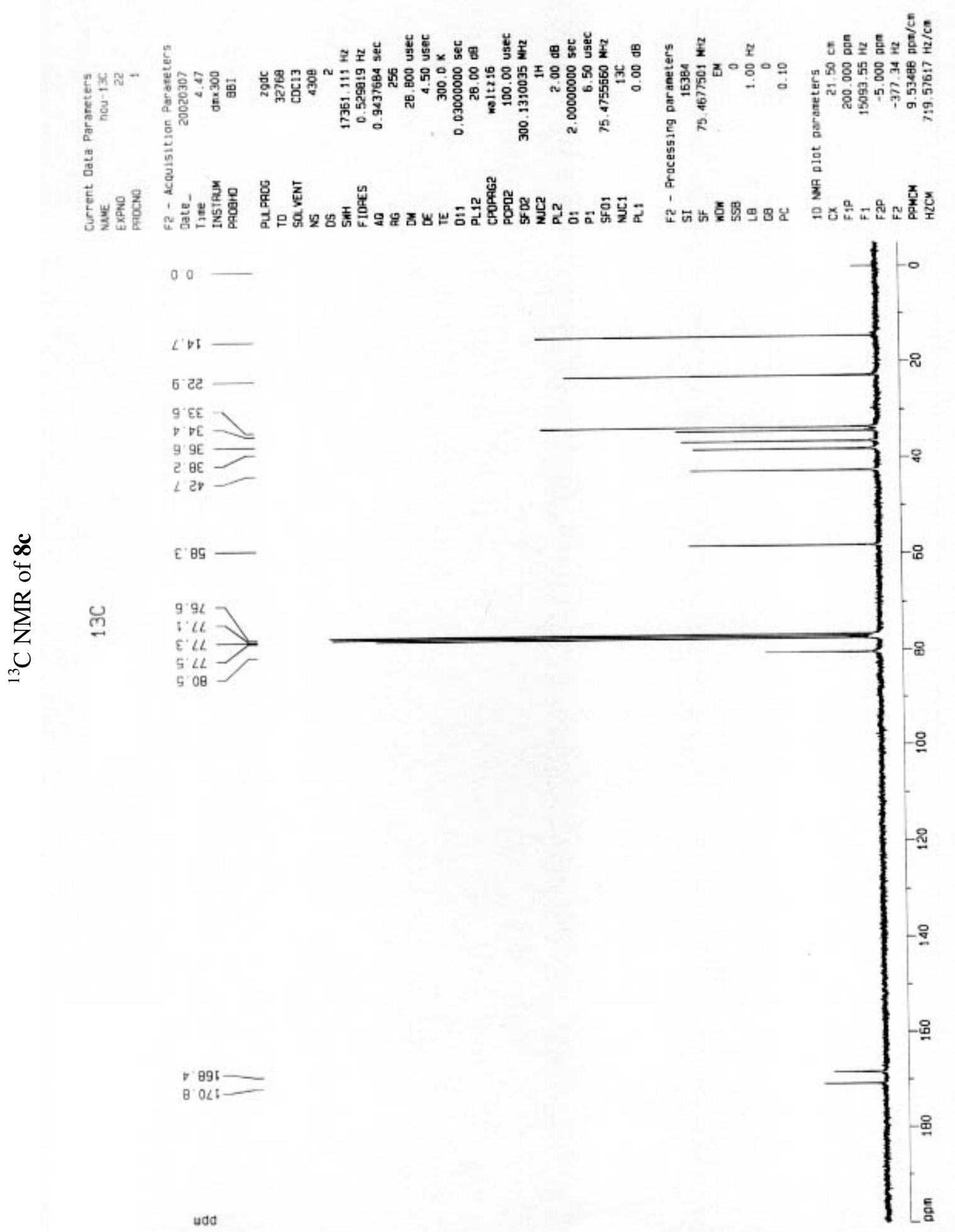

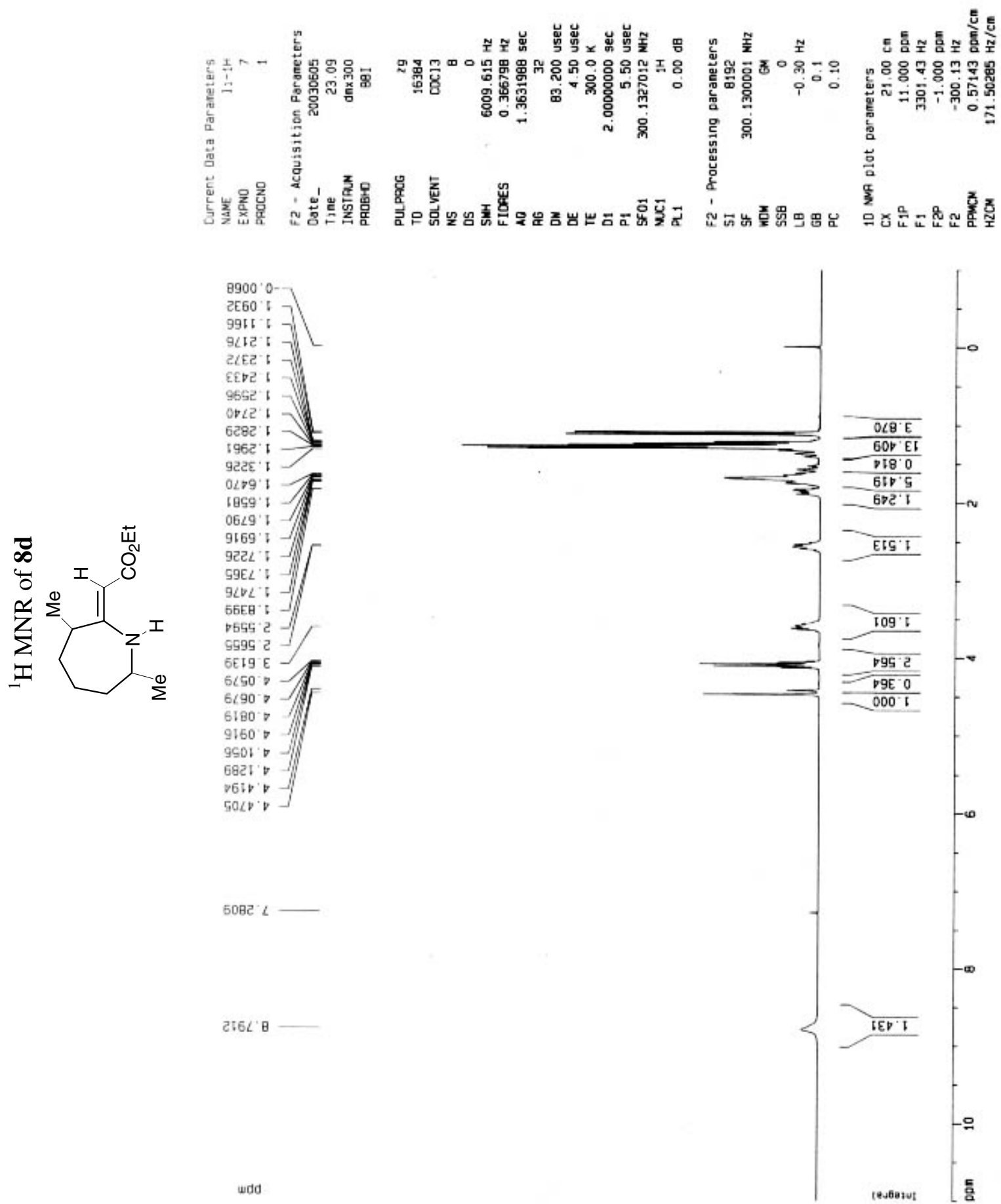

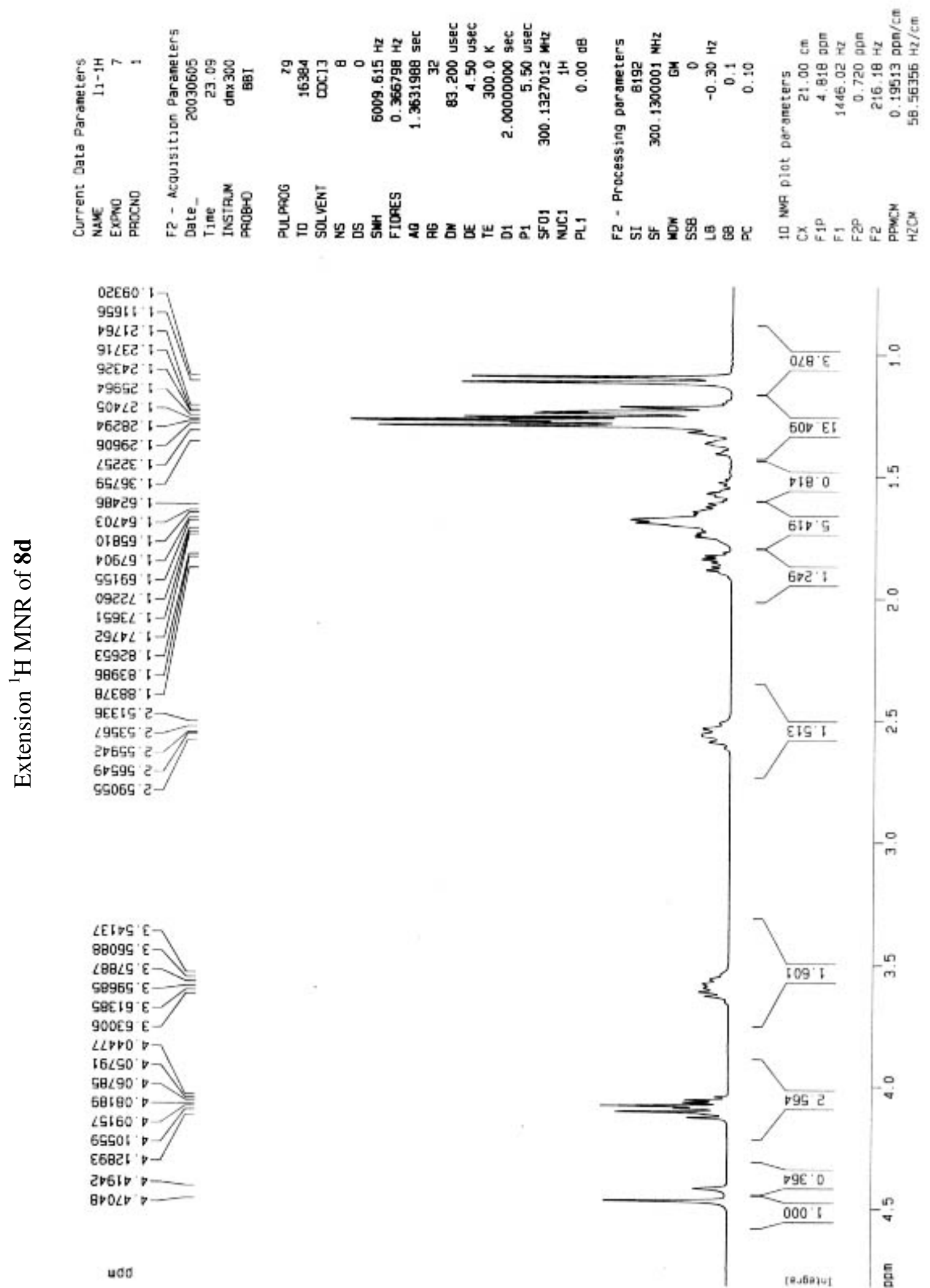


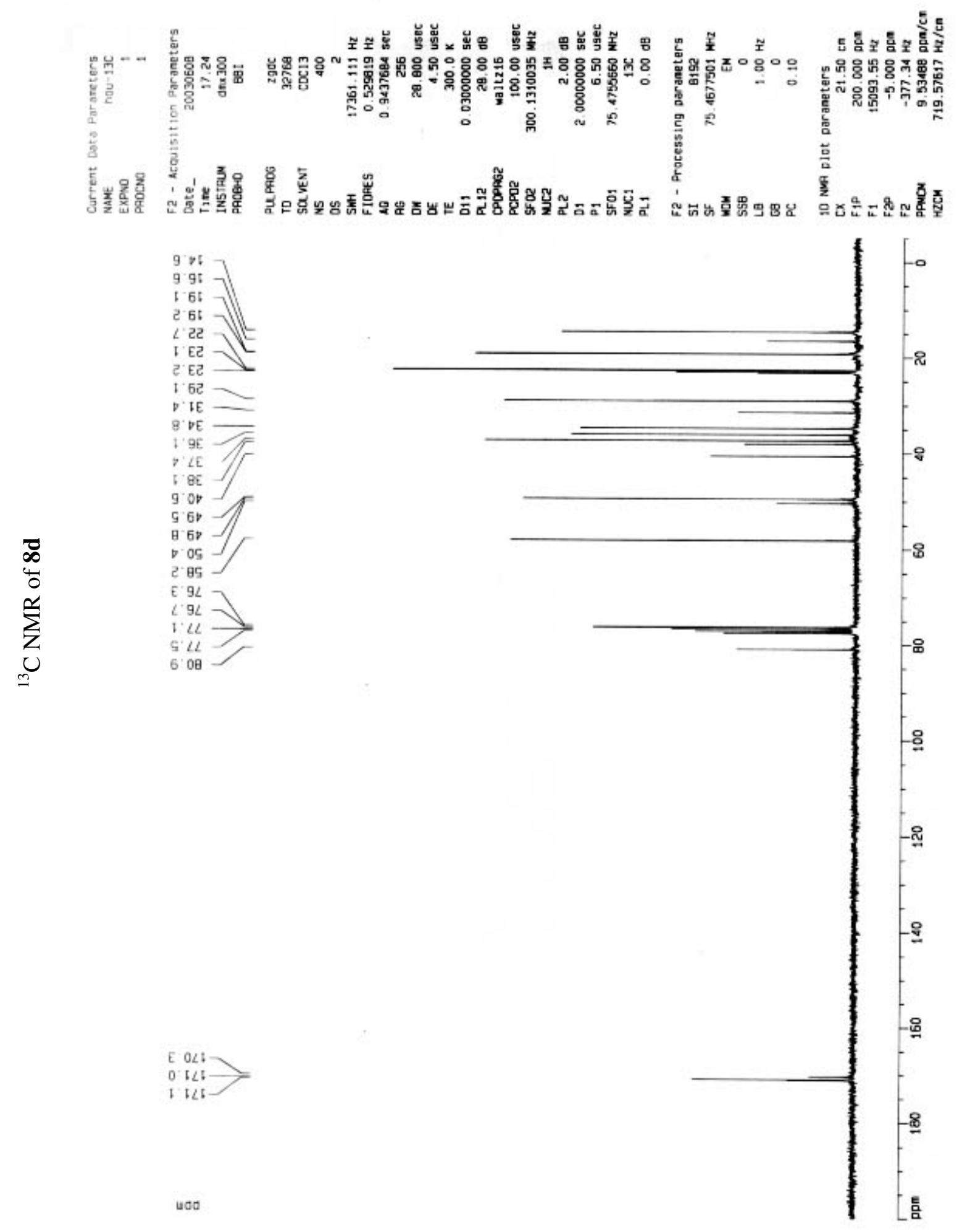




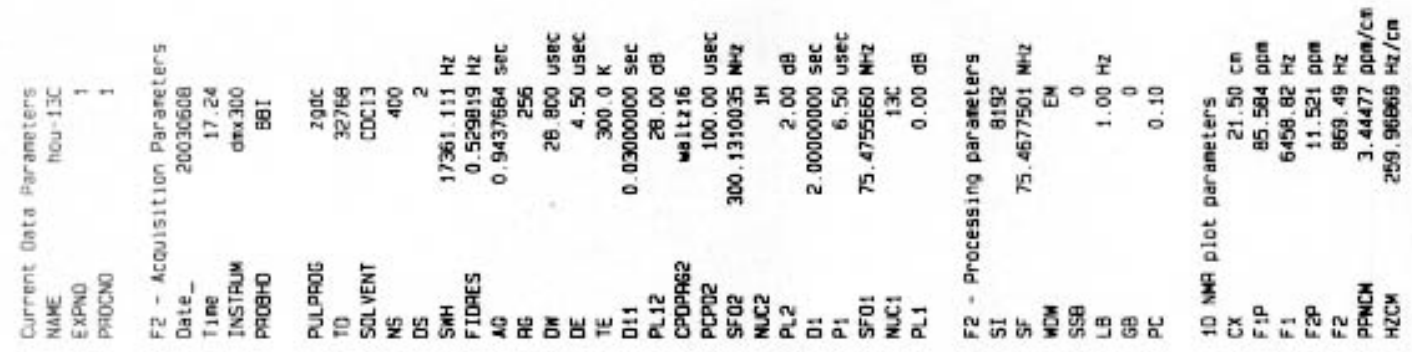

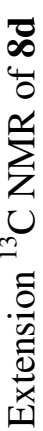
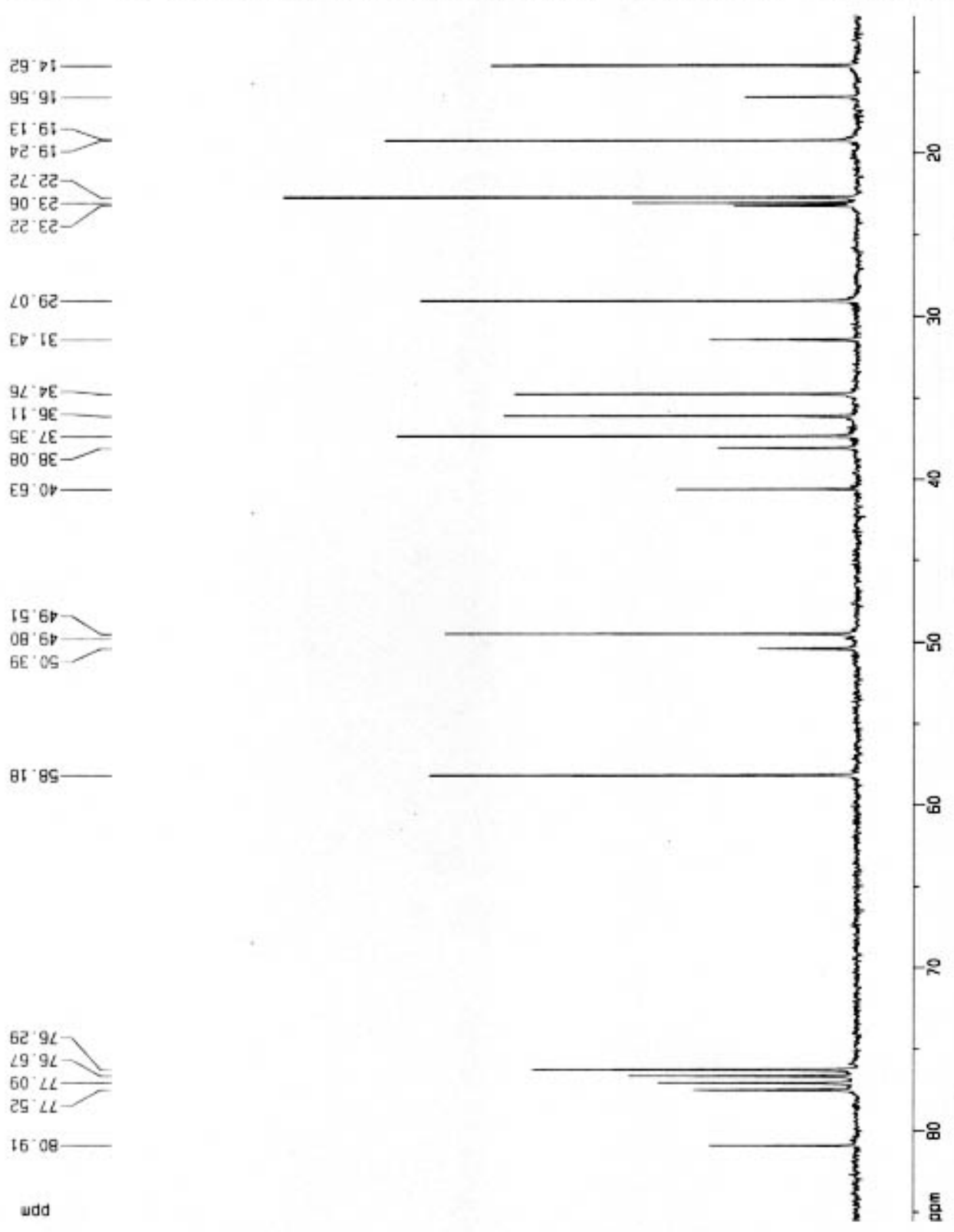

$\angle 0^{\circ} 62$

EV IE

$9 L^{\circ}>E$

II $98-\square$

$80^{\circ} \mathrm{BE}-$

69. $6 b$
$00^{\prime} 6 \mathrm{~b}$

$6 E^{\circ}$ OS

B' $8 \mathrm{~S}^{-}$

udd 